\title{
Illumination Waveform Optimization for Time-of-Flight Range Imaging Cameras
}

\author{
A.D. Payne*, A.A. Dorrington, and M.J. Cree \\ University of Waikato, Private Bag 3105, Hamilton 3240, New Zealand
}

\begin{abstract}
Time-of-flight range imaging sensors acquire an image of a scene, where in addition to standard intensity information, the range (or distance) is also measured concurrently by each pixel. Range is measured using a correlation technique, where an amplitude modulated light source illuminates the scene and the reflected light is sampled by a gain modulated image sensor. Typically the illumination source and image sensor are amplitude modulated with square waves, leading to a range measurement linearity error caused by aliased harmonic components within the correlation waveform. A simple method to improve measurement linearity by reducing the duty cycle of the illumination waveform to suppress problematic aliased harmonic components is demonstrated. If the total optical power is kept constant, the measured correlation waveform amplitude also increases at these reduced illumination duty cycles.

Measurement performance is evaluated over a range of illumination duty cycles, both for a standard range imaging camera configuration, and also using a more complicated phase encoding method that is designed to cancel aliased harmonics during the sampling process. The standard configuration benefits from improved measurement linearity for illumination duty cycles around $30 \%$, while the measured amplitude, hence range precision, is increased for both methods as the duty cycle is reduced below $50 \%$ (while maintaining constant optical power).
\end{abstract}

Keywords: Time of flight, range imaging, range camera, aliasing, harmonic cancellation, linearity, precision.

\section{INTRODUCTION}

Time-of-flight range imaging cameras produce an image in which every pixel contains both intensity and distance (range) information. These cameras typically measure range with an active light source, using the amplitude modulated continuous wave (AMCW) technique to determine time-of-flight simultaneously for every pixel in the image. Acquisition of a range image can therefore be performed at a rapid update rate, making range imaging cameras suitable for a variety of applications including natural human user interfaces, automotive safety and control, machine vision, and medical imaging.

A constant demand exists to improve these cameras and to provide enhanced measurement accuracy, precision and resolution, while also reducing cost and power consumption. One problem that these cameras face is that they often suffer from a measurement linearity error due to the use of non-sinudoidal amplitude modulated waveforms ${ }^{1}$. A number of studies have focused on correcting this error during post processing, by modeling or calibrating the system ${ }^{1-6}$. This paper instead investigates how the shape of the amplitude modulated illumination waveform contributes to the range measurement linearity and precision, and how this waveform shape can be optimized to improve these attributes.

\section{MEASUREMENT TECHNIQUE}

Range measurements are performed by illuminating the entire scene with an amplitude modulated light source. The light is reflected from objects in the scene back to an image sensor, where the propagation time is manifested as a phase shift of the modulation envelope. Each pixel within the image sensor is gain modulated with a reference signal at the same frequency as that of the light source (homodyne detection), producing a correlation between the received illumination and image sensor (reference) waveforms. With objects at a short range from the camera, the returned illumination and image sensor modulation signals are in phase, producing a bright pixel value; while for longer ranges, the pixel

*a.payne@waikato.ac.nz; phone +64 7 838-4841; fax +64 7 838-4835; eng.waikato.ac.nz

Videometrics, Range Imaging, and Applications XI, edited by Fabio Remondino, Mark R. Shortis, Proc. of SPIE Vol. 8085, 80850D - @ 2011 SPIE · CCC code: 0277-786X/11/\$18 · doi: 10.1117/12.889399 
brightness decreases as the increased propagation time induces a phase shift in the illumination signal's modulation envelope.

As the pixel brightness is dependent not only on an object's range, but also on its color or reflectivity and the level of ambient light, multiple correlation measurements are taken to determine three unknowns; the amplitude $A$, offset $B$, and phase $\varphi$. Typically the correlation waveform is sampled four times by electronically stepping the illumination signal phase by $90^{\circ}$ between each sample, producing four intensity measurements $I_{0}$ to $I_{3}$. The unknown parameters are then calculated as

$$
\begin{gathered}
A=\frac{1}{2} \sqrt{\left(I_{1}-I_{3}\right)^{2}+\left(I_{0}-I_{2}\right)^{2}}, \\
B=\frac{1}{4} \sum_{k=0}^{3} I_{k}, \\
\varphi=\operatorname{atan} 2\left(I_{0}-I_{2}, I_{1}-I_{3}\right) .
\end{gathered}
$$

From the measured phase, the object range is then calculated for each pixel as

$$
r=\frac{\varphi c}{4 \pi f},
$$

where $c$ is the speed of light and $f$ is the modulation frequency of the illumination source and image sensor modulation waveforms (typically of the order of 10-100 MHz).

\subsection{Measurement linearity}

The phase measurement in equation (3) assumes that the sampled correlation waveform values follow a sinusoidal shape. Hence it may seem sensible to modulate the illumination source (or image sensor) with a sinusoidal waveform, but this is not usually possible. In practice, the light source used for most range imaging cameras typically consists of a bank of infrared light emitting diodes (LEDs) that are controlled by digitally generated square wave signals. The limited electronic bandwidth of the LEDs means that the optical output actually lies somewhere between a square wave and a sinusoid $^{4}$. Similarly, the image sensor is driven by a digitally generated square wave. These waveforms therefore contain higher order harmonic components.

If both the illumination and image sensor waveforms contain harmonic components of the same order, the correlation waveform will also contain harmonic components (from the correlation theorem). When the correlation waveform is sampled, harmonics of order $h=i N \pm 1$ are aliased onto the fundamental frequency, where $N$ is the total number of intensity measurements used and $i$ is the set of positive integers ${ }^{7}$. These aliased harmonics corrupt the phase measurement in equation (3), resulting in a periodic systematic error as illustrated in Figure 1. This phase error therefore produces a corresponding range measurement error, as follows from equation (4).

The phase linearity error is typically corrected by performing a calibration of the system, and applying compensation during post processing using a look up table or parametric fit ${ }^{1-5}$. There are two major limitations to this approach; firstly the calibration process can become time consuming, particularly if a per-camera or even a per-pixel correction is required $^{5,8}$, and secondly, the correction is only valid for the conditions under which the calibration was performed. If environmental factors (such as temperature) or system parameters (such as the modulation frequency) are altered then the effectiveness of the correction is likely to be reduced. Linder et al. modeled the correlation waveform as a combination of a sinusoid and triangular waveform, and combined phase measurements from each ${ }^{6}$. However, an appropriate weighting function is found through calibration, hence the method still suffers from the limitations described above.

As the linearity error results from aliased harmonic components of order $h=i N \pm 1$, this error can be avoided (or reduced) by increasing $N$, the number of samples of the correlation waveform ${ }^{4,9}$. Since this approach does not rely on any calibration parameters, it is more robust to environmental or system changes, however, the measurement acquisition time is somewhat proportional to $N$ (due to additional image sensor readout periods), so this method is seldom used as it also increases motion blur problems for dynamic scenes. 


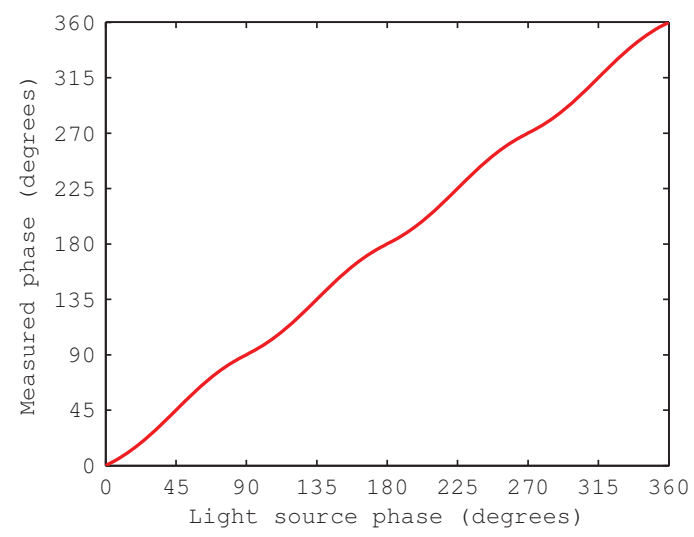

Figure 1. Simulated phase linearity where both the illumination source and image sensor respond perfectly to modulation with square waveforms.

Another approach cancels the aliased harmonic components during the sampling process ${ }^{10}$. To achieve this cancellation, a phase modulation pattern is applied to the light source signal during the sensor integration period, and the aliased harmonics are summed together destructively. By suppressing the aliased harmonics, the measurement linearity is again robust to environmental or system changes, while the acquisition time is not extended as only four samples of the correlation waveform are required. While this approach does add a small amount of complexity to the signal generation electronics, the main disadvantage is that the amplitude of the fundamental frequency component is reduced, effecting measurement precision as discussed in the following section.

\subsection{Measurement precision}

For an ideal system that is limited by photon shot noise, measurement precision (standard deviation) is primarily dictated by four parameters ${ }^{11}$, namely the modulation frequency $f$, demodulation contrast $c_{d}$, and the measured offset from the active and ambient illumination sources, denoted $B_{\text {active }}$ and $B_{\text {ambient }}$ respectively. The measured offset $B$ from equation (2) is the sum of these two parameters, therefore $B=B_{\text {active }}$ in the absence of ambient light. The measurement precision (standard deviation) is given by

$$
\sigma_{\mathrm{r}}=\frac{c}{4 \pi f \sqrt{2}} \cdot \frac{\sqrt{B_{\text {ambient }}+B_{\text {active }}}}{c_{d} B_{\text {active }}},
$$

where the demodulation contrast is given by

$$
c_{d}=\frac{A}{B_{\text {active }}} \text {. }
$$

The modulation frequency $f$, demodulation contrast $c_{d}$, and active signal offset $B_{\text {active }}$ are dependent on the system configuration and therefore can be altered to improve the resultant range measurement precision. One simple approach is to increase the total optical power emitted (and hence received), increasing $B_{\text {active, }}$ but this is also likely to increase power consumption, generated heat, and the cost of the system.

Increasing the modulation frequency provides an effective means of improving range measurement precision $^{12}$, however it also reduces the maximum unambiguous measurement range of the system ${ }^{1}$, given as $c /(2 f)$. Objects beyond this range introduce ambiguity as the measured phase shift in equation (3) is bound to $[-\pi, \pi)$ radians. Techniques, such as combining multiple phase measurements from different modulation frequencies, can be used to overcome this ambiguity problem $^{13}$, although they also introduce additional complexity and processing to the system.

The third parameter, demodulation contrast, should have a value close to 1 in an ideal system. There are a number of factors that can reduce this demodulation contrast value, including the natural sampling process used, imperfect modulation of the illumination source, or imperfect charge separation within the image sensor pixels ${ }^{1,14}$. If no harmonic components are aliased on to the fundamental frequency component during sampling, the demodulation contrast is simply given by the amplitude of the fundamental frequency component of the correlation waveform divided by the DC 
offset (assuming no ambient light) as given in equation(6). Both of these parameters are constant, thus the demodulation contrast $c_{d}$ is also constant.

In the case where aliasing occurs, the aliased harmonics are added to the fundamental frequency component being measured, producing (phase dependent) constructive or destructive interference. The measured amplitude $A$, hence demodulation contrast, is therefore also phase dependent as shown in Figure 2 for square wave modulation of the illumination and image sensor. In this paper the mean value of the demodulation contrast over the 0 to $360^{\circ}$ range is considered to be a suitable metric to evaluate the resultant precision for a system that suffers from this phase dependency.

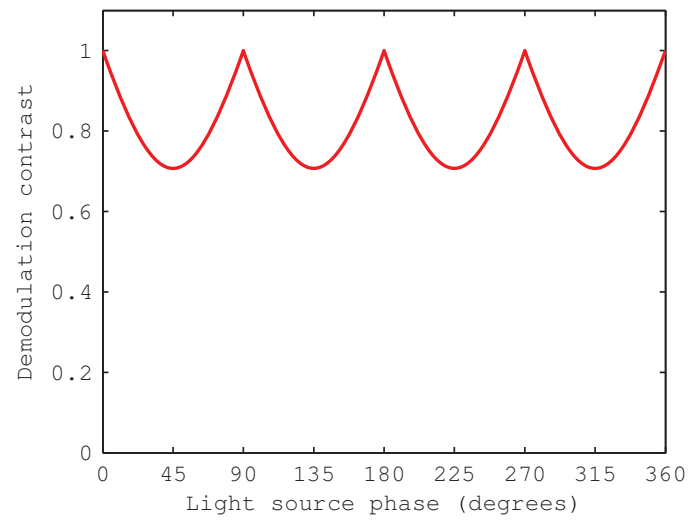

Figure 2. Simulated demodulation contrast when both the illumination and image sensor are modulated with a square wave. The value is phase dependent due to the constructive/destructive addition of aliased harmonics.

\section{INCREASING THE DEMODULATION CONTRAST}

For a system where the illumination source is amplitude modulated with a sinusoid and the image sensor is modulated with a rectangular waveform, due to the natural sampling process, the maximum possible demodulation contrast value can be expressed as ${ }^{14}$

$$
c_{d, \max }=\operatorname{sinc}(\pi d)
$$

where $d$ is the duty cycle of the image sensor modulation waveform. Therefore, for $50 \%$ duty cycle square wave modulation, $c_{d, \max }$ is limited to 0.64 , whereas at a $25 \%$ duty cycle this value is increased ${ }^{14}$ to 0.9 . The demodulation contrast, hence measurement precision, can therefore be improved by reducing the duty cycle of the image sensor modulation waveform.

In practice, most range imaging systems utilize a specialized CCD/CMOS image sensor to provide the high frequency gain modulation at each pixel. These image sensors typically employ what is known as a 2-tap pixel structure ${ }^{1,8,14}$, producing two correlation measurements simultaneously that are $180^{\circ}$ out of phase. This improves the efficiency of the system as the received light that would otherwise be discarded during the modulation low or 'off' state now contributes to the complementary measurement. The complementary nature of the pixel design however restricts these sensors to use a $50 \%$ duty cycle waveform. It is possible to create 4-tap pixels that produce all four correlation measurements simultaneously, thereby utilizing $25 \%$ duty cycle modulation. However, 4-tap pixel designs can suffer from lower fill factors and increased fixed pattern noise, so 2-tap pixel structures are generally preferred ${ }^{14}$.

While equation (7) is based on the use of sinusoidal illumination modulation, most practical systems utilize digitally generated square wave modulation for the illumination source. The concept of reducing the duty cycle can therefore instead be applied to the illumination source to avoid the restrictions imposed by the image sensor. A simulation of the resulting demodulation contrast is shown in Figure 3 for different illumination duty cycles, while the image sensor modulation is fixed at a duty cycle of $50 \%$. The phase dependency described in section 2.2 causes the measured demodulation contrast value to span a range of values at each particular illumination duty cycle, shown by the shaded region in Figure 3, while the mean value is plotted as a solid line. The figure shows that the mean demodulation contrast 
value increases for decreased illumination duty cycles, and that the measured demodulation contrast can exceed a value of 1 in the presence of aliased harmonics (using the definition of $c_{d}$ given in equation (6)).

Figure 3 also incorporates a simulation of the measured demodulation contrast when applying the harmonic cancellation technique described by Payne et al. ${ }^{10}$ to the same system. As the aliased harmonic components are removed during the sampling process, the demodulation contrast value is constant for each illumination duty cycle, and therefore appears as a line in the plot (there is no corresponding shaded region). The demodulation contrast values are lower than those of the corresponding homodyne mode of operation, approaching a maximum value of 1 as the illumination duty cycle is decreased. As there are no additional contributions from aliased harmonics in this mode of operation, the demodulation contrast value conforms to that given in equation (7).

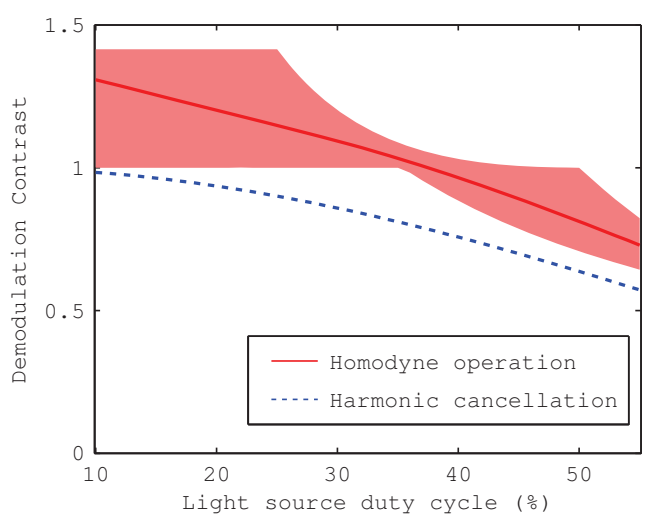

Figure 3. Simulated demodulation contrast where the image sensor is modulated with a fixed $50 \%$ duty cycle square wave and the illumination source is modulated with a rectangular waveform. The shaded region indicates the phase dependent values and the solid line is the mean value over the range 0 to $360^{\circ}$.

\subsection{Change in range measurement linearity}

Reducing the illumination duty cycle to increase the demodulation contrast also has a secondary effect of altering the harmonic content of the correlation waveform, thereby affecting range measurement linearity. A simulation of the measured phase was performed for different illumination duty cycles, with the resultant RMS phase linearity error shown in Figure 4. For the standard homodyne method of operation, it can be seen that the linearity error is minimized at a duty cycle of approximately $29 \%$.

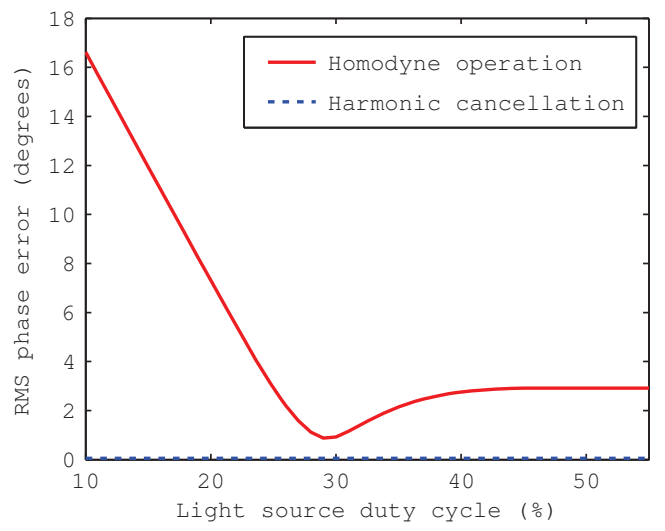

Figure 4. Simulated RMS phase linearity error where the image sensor is modulated with a $50 \%$ duty cycle square wave and the illumination source is modulated with a rectangular waveform.

The rectangular illumination and sensor modulation waveforms can be written using a Fourier series as

$$
x_{\text {rect }}(t)=d+\frac{2}{\pi} \sum_{h=1}^{\infty} \frac{\sin (\pi h d)}{h} \cdot \cos (2 \pi h f t),
$$


where $d$ is the duty cycle (between 0 and 1 ), and $f$ is the modulation frequency. The spectrum of the resultant correlation waveform can then be determined using the correlation theorem

$$
F\{g \otimes h\}=(F\{g\})^{*} \cdot F\{h\}
$$

where $\Phi$ denotes the Fourier transform, $\otimes$ is the correlation function, and $*$ denotes the complex conjugate.

When the correlation waveform is sampled four times $(N=4)$, odd order harmonics are aliased onto the fundamental frequency component as described in section 2.1. Of these harmonics, as can be determined from equations (8) and (9), the third order harmonic generally has the largest amplitude, and therefore has the greatest contribution to the phase linearity error. As the duty cycle of the rectangular illumination waveform approaches $33.3 \%$, the amplitude of this third harmonic approaches zero, and the RMS error is reduced. However, at this point the amplitude of the next aliased (fifth order) harmonic becomes significant. In the simulation in Figure 4, the RMS phase linearity error is minimized at a duty cycle of approximately $29 \%$, when the third and fifth order harmonics are destructively added together, at which point further higher order odd harmonic components also become significant.

\subsection{Emitted optical power}

Reducing the duty cycle of the illumination waveform produces a proportional decrease of the average emitted optical power, hence the measured active offset value $B_{\text {active. }}$. While this $B_{\text {active }}$ term reduces with duty cycle, when determining the resultant range measurement standard deviation using equation (5), it competes against the increasing demodulation contrast $c_{d}$. In the absence of ambient light, the measurement standard deviation is therefore not expected to worsen at duty cycles above $25 \%$ (compared to the standard deviation of a system using a $50 \%$ duty cycle waveform), and can actually improve at duty cycles around $37 \%$, despite the decrease in emitted optical power.

Alternatively, the average emitted optical power can be maintained by inversely scaling the peak power against duty cycle. For example, a 25\% duty cycle waveform emitting twice the peak optical power (for half of the duration) will achieve the same average optical power as a $50 \%$ duty cycle waveform.

\section{EXPERIMENTAL SETUP}

A Canesta XZ422 time of flight range imaging camera was employed to verify the advantages of reducing the illumination source duty cycle. The camera provides a resolution of $160 \times 120$ pixels, operates at a default modulation frequency of $44 \mathrm{MHz}$, and uses a bank of 20 infrared (870nm) LEDs as the illumination source.

For the first experiment two significant modifications were made to the camera configuration. First, due to the limited rise and fall times of the amplitude modulated LEDs, the illumination source was replaced with an external laser diode. Second, a field programmable gate array (FPGA) was used to introduce programmable phase shifts and duty cycle changes to the light source modulation signal before it reached the laser. A photo of the setup is shown in Figure 5.

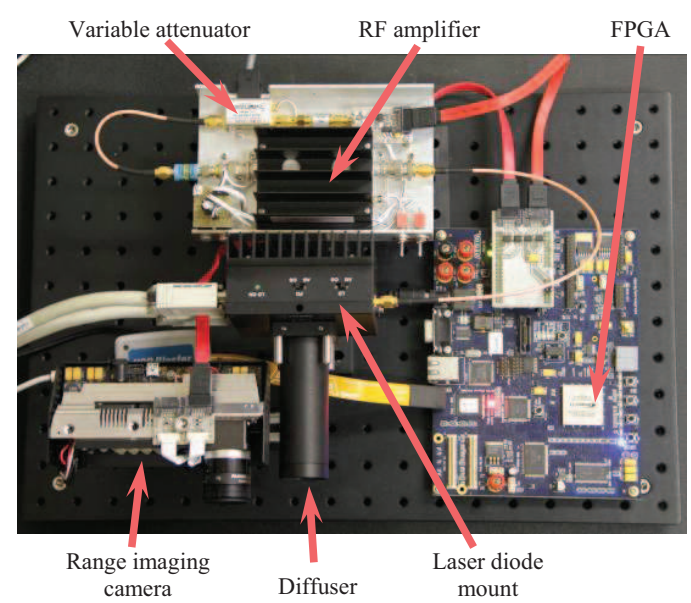

Figure 5. Photograph of the XZ422 range imaging camera connected to a FPGA and laser illumination source. 
The illumination modulation signal that normally connects to the LED array is first routed to a phase locked loop (PLL) within a Stratix II FPGA (Altera Corp., CA, USA), allowing the signal phase and duty cycle to be controlled in real time. The signal is then passed through a variable attenuator and RF amplifier (ZX76-31R5 and ZPUL-30P respectively, MiniCircuits, NY, USA) to a temperature controlled laser mount (LTC100-B, Thorlabs Inc., NJ, USA) where it modulates the drive current of a laser diode. An $850 \mathrm{~nm} 100 \mathrm{~mW}$ laser diode illuminates the scene through a ground glass diffuser.

The average optical output power was kept constant, and the experiments were performed in a dark environment, due to the fact that the demodulation contrast cannot be directly measured using the XZ422 range imaging camera (discussed in more detail in section 4.2). The laser output was measured using a $2 \mathrm{GHz}$ photodiode (SV2-FC, Thorlabs Inc.), and the RF modulation and DC levels were adjusted to suitable levels at each duty cycle using the variable attenuator and laser controller.

The FPGA was programmed to operate in two operating modes; a standard homodyne mode, and a harmonic cancellation mode. The harmonic cancellation mode shifts the phase of the illumination signal at predetermined times within the camera integration period to cancel aliased harmonics from the correlation waveform ${ }^{10}$. In this instance the programmed configuration provided cancellation of odd harmonics up to and including 9th order.

To measure the demodulation contrast and phase linearity error, a flat target was placed in front of the camera. Instead of moving the target to different distances, an electronic delay was added to the illumination signal to shift the phase. This approach has the advantage that the optical power from the laser diode can be (relatively) low compared to the camera LED array as the target remains close to the camera, electronic phase shifts can be quickly and precisely set, multipath effects within the scene can be avoided, and the illumination power reflected back to the camera remains constant, as opposed to decreasing with target distance, thereby avoiding any errors that may be related to changes in signal intensity.

\subsection{Measured linearity error}

An example of the measured linearity error is shown in Figure 6 (a), where 40 electronic phase shifts have been used over the 0 to $360^{\circ}$ range. In this particular capture, the laser duty cycle is set to $25 \%$. Each data point gives the difference between the known electronic phase and an average of the measured phase over $20 \times 20$ pixels in the center of the image. A linear interpolation between the measured data points is included to visually enhance the figure. The harmonic cancellation mode can be seen to significantly reduce the systematic oscillatory error, despite all other system parameters remaining constant.

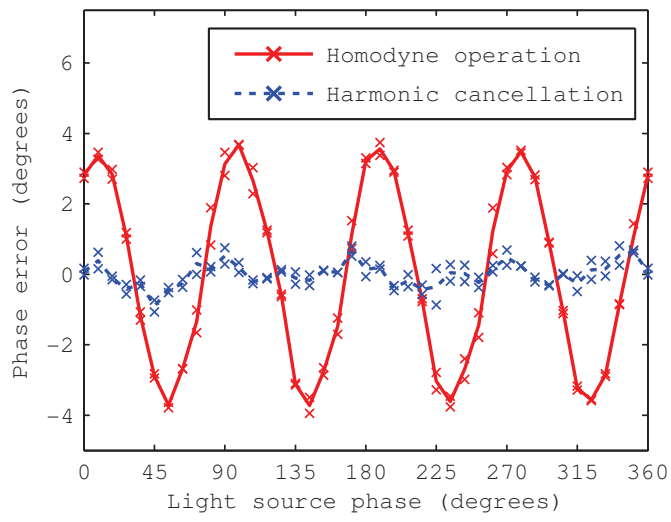

(a)

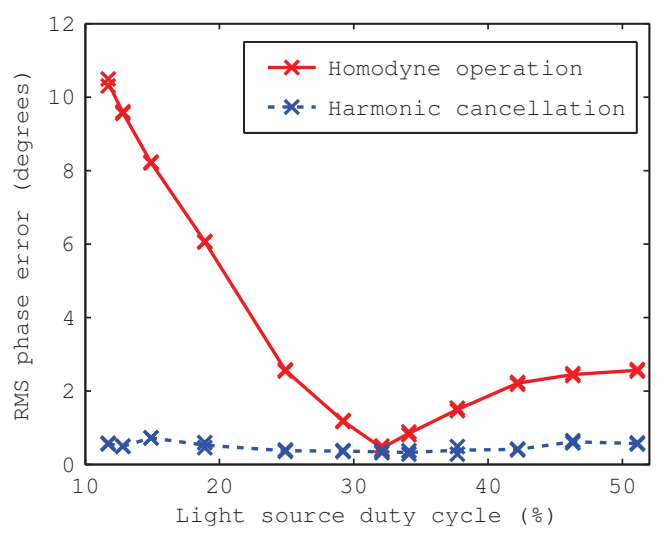

(b)

Figure 6. (a) Example of the measured phase linearity error when the laser light source is modulated with a $25 \%$ duty cycle rectangular wave. (b) Measured RMS phase linearity error where the image sensor is modulated with a $50 \%$ duty cycle square wave and the laser illumination source is modulated with a rectangular waveform of varied duty cycle.

The phase linearity error measurement was performed using rectangular illumination waveforms with duty cycles spanning 12 to $52 \%$, in each case maintaining a constant average optical power. The measured RMS error is shown in Figure 6 (b). With the harmonic cancellation mode enabled, the systematic oscillatory error introduced by aliased harmonics is significantly reduced; hence the measured RMS error is largely independent of the duty cycle of the 
illumination source. The measured RMS error has an offset of approximately $0.5^{\circ}$ in all of the harmonic cancellation measurements, which can be attributed to a combination of imperfect cancellation of the harmonic components and other random noise sources in the system.

In the standard operating mode, the measured RMS error approximately follows the shape of the simulated error from Figure 4. In general, the measured error is smaller than that in the simulation, with the minimal measured linearity error occurring at a duty cycle of $32 \%$, compared to $29 \%$ in the simulation. This discrepancy is primarily due to electronic bandwidth limitations of the image sensor, as the modulation waveform is not perfectly square; hence the aliased harmonics are partially attenuated and contribute smaller error than in the simulation.

\subsection{Measured demodulation contrast}

Most 2-tap range imaging sensors, including the Canesta XZ422, include additional electronics within each pixel to suppress ambient light to avoid pixel saturation, for example refer ${ }^{14}$. A single value is output from each pixel consisting only of the difference between the two simultaneous correlation measurements, e.g. $I_{0}-I_{2}$. While this feature removes the offset due to ambient light $B_{\text {ambient }}$, it also removes the offset due to the active light source $B_{\text {active }}$, therefore it is not possible to measure the demodulation contrast using equation (6) with this camera.

To overcome this limitation, it is assumed that the correlation waveform offset $B$ remains constant throughout the experiment. This is a reasonable assumption due to the fact that the average emitted illumination power is set to a constant value regardless of the duty cycle used; while the reflected illumination also remains constant since the physical target is stationary (and the phase shift is added electronically), and ambient light is avoided. Under these conditions, the demodulation contrast is therefore proportional to the measured amplitude $A$, and can be measured by the image sensor.

The measured amplitude for each illumination duty cycle is plotted in Figure 7 (in camera specific amplitude units). Since the amplitude is phase dependent in the presence of aliased harmonics as described in section 2.2, 40 offsets over the 0 to $360^{\circ}$ range were again electronically added to the illumination signal. Each of the amplitude measurements is plotted using a cross marker, and a line is also plotted between the mean value at each duty cycle. For the standard operating mode, the spread of the points for duty cycles less than $30 \%$, or greater than $40 \%$, are therefore largely due to the systematic phase dependency (as opposed to measurement noise).

In the standard mode of operation, the phase dependent amplitude variation is smaller than that predicted in the demodulation contrast simulation in Figure 3 due to the fact that the image sensor modulation waveform is not perfectly square as mentioned in section 4.1. The measured data still show the same attributes as the simulation however, where the mean amplitude is increased for shorter duty cycles, and the spread of points is minimized at the $34 \%$ duty cycle measurement (35\% in the simulation).

In the harmonic cancellation mode the amplitude values are closely bunched together, primarily limited by measurement noise rather than a systematic oscillation. Again, the mean amplitude increases for shorter duty cycles, with the change in close agreement with that simulated.

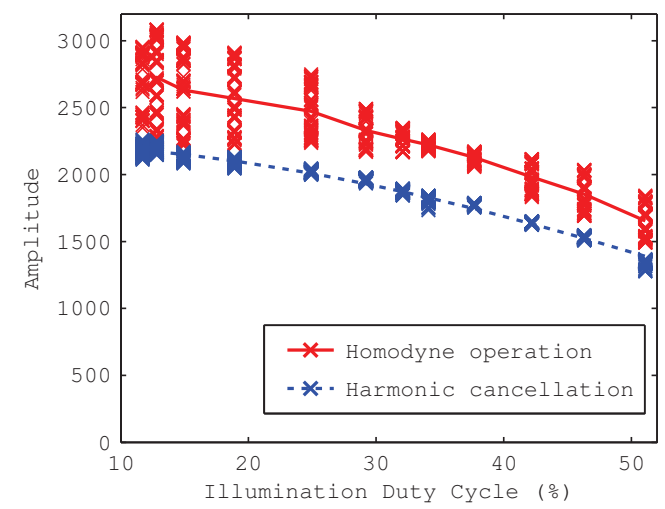

Figure 7. Measured amplitude for a rectangular laser illumination waveform at different duty cycles (with a constant average optical power). 


\section{OPTIMIZING LED MODULATION}

As most existing range imaging cameras utilize a LED illumination source, the effectiveness of altering the illumination duty cycle was evaluated using the standard Canesta XZ422 range camera hardware, without the laser and FPGA modification described above, and also in the presence of ambient light. Figure 8 shows a photo of the setup, where a flat target was fixed to a linear translation stage and the target was moved in small increments while the range and amplitude were measured at each position. To minimize measurement errors due to multi-path scattering within the scene, where the emitted light is reflected from multiple objects before returning to the camera, the background was covered with a black material and the flat target was covered with a retro-reflective vinyl sheet (3M, MN, USA). This configuration ensured that the target under measurement is significantly brighter than other (potentially interfering) objects within the scene.

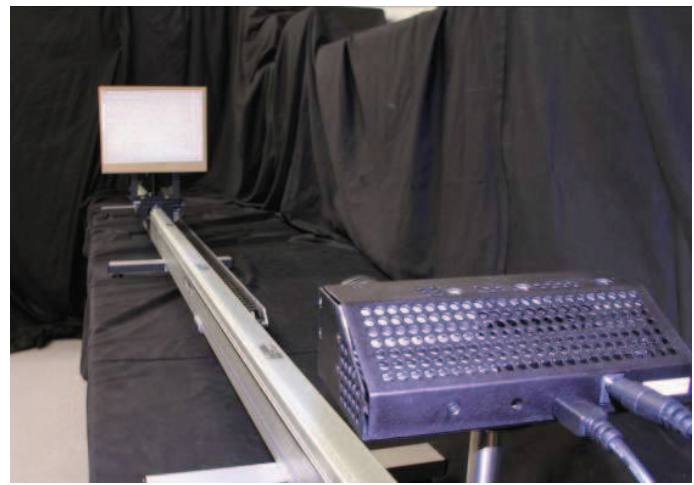

Figure 8. Linear translation stage with retro-reflective target used to evaluate range camera linearity.

Using the XZ422 camera, the LED drive current amplitude and pulse width can be altered by modifying the range camera firmware. A photodiode was placed in front of the LEDs, and these parameters were adjusted to provide three different waveforms for a modulation frequency of $22 \mathrm{MHz}$ as shown in Figure 9(a); a $50 \%$ duty cycle waveform approximating normal square wave operation, a waveform with priority given to minimizing the $3^{\text {rd }}$ harmonic, and a waveform attempting to minimize both the $3^{\text {rd }}$ and $5^{\text {th }}$ harmonics. For the modified waveforms an effort was made to match the mean optical power of the square wave, measured using a laser power meter (PM120D, Thorlabs Inc., NJ, USA). The measured waveform parameters are given in Table 1 , where the amplitudes of the $3^{\text {rd }}$ and $5^{\text {th }}$ order harmonics are given relative to the amplitude of the fundamental frequency component. As the unmodified camera hardware cannot shift the phase of the illumination signal during the integration period, the harmonic cancellation technique was excluded from these tests.

The linear translation stage was moved in $1 \mathrm{~cm}$ increments over a $3.41 \mathrm{~m}$ distance, corresponding to $\pi$ radian phase shift at the $22 \mathrm{MHz}$ modulation frequency, and the range and amplitude was measure for a region of $10 \times 16$ pixels - defined as the target size at the farthest distance. The mean measured range value was subtracted from the known target range to produce a linearity error, shown in Figure 9 (b). The measured amplitude, calculated using (1), is given in Figure 9 (c), along with the normalized amplitude error in Figure 9 (d).

As anticipated, the $50 \%$ duty cycle waveform produces a large oscillatory linearity error (108 mm peak to peak); whereas the linearity errors from the modified waveforms are significantly smaller $(30 \mathrm{~mm}$ and $21 \mathrm{~mm}$ peak to peak respectively). The shape of an error waveform provides information as to which aliased harmonic components are dominant, where the $3^{\text {rd }}$ and $5^{\text {th }}$ order harmonics generate four oscillations over a $2 \pi$ radian phase shift; the $7^{\text {th }}$ and $9^{\text {th }}$ order harmonics generate eight oscillations, etc. The $50 \%$ duty cycle waveform (red $\times$ ) shows two large oscillations over the distance corresponding to a $\pi$ radian phase shift in Figure 9 (b), primarily generated by the $3^{\text {rd }}$ order harmonic. When the $3^{\text {rd }}$ order harmonic is minimized $\left(\right.$ green + ) the $5^{\text {th }}$ order harmonic becomes dominant, producing two oscillations in the linearity error plot of Figure 9 (b), however the oscillation in the amplitude error plot of Figure 9 (d) is inverted compared to that of the $50 \%$ duty cycle waveform. Finally, by attempting to minimize both the $3^{\text {rd }}$ and $5^{\text {th }}$ order harmonics (blue ·), the linearity error in Figure 9 (b) shows a combination of both two and four oscillations over the measured distance caused by a combination of $3^{\text {rd }}, 5^{\text {th }}, 7^{\text {th }}$, and possibly $9^{\text {th }}$ order harmonics. This result illustrates the limiting factor of this approach; as the waveform is altered to minimize one particular harmonic component, other harmonics become dominant and the error cannot be completely removed. 


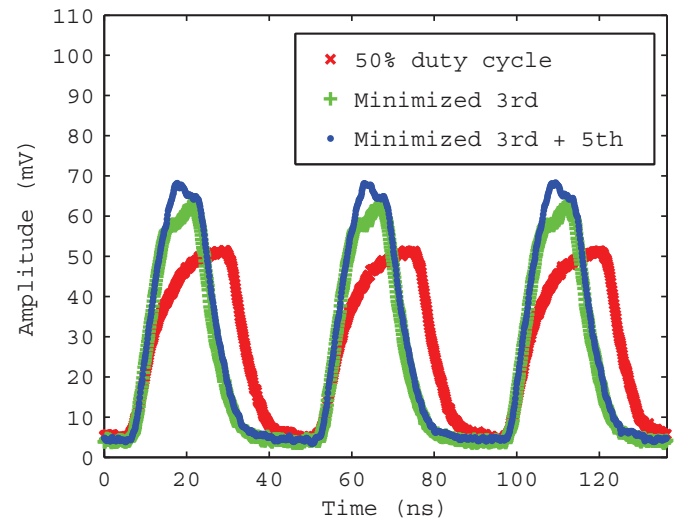

(a)

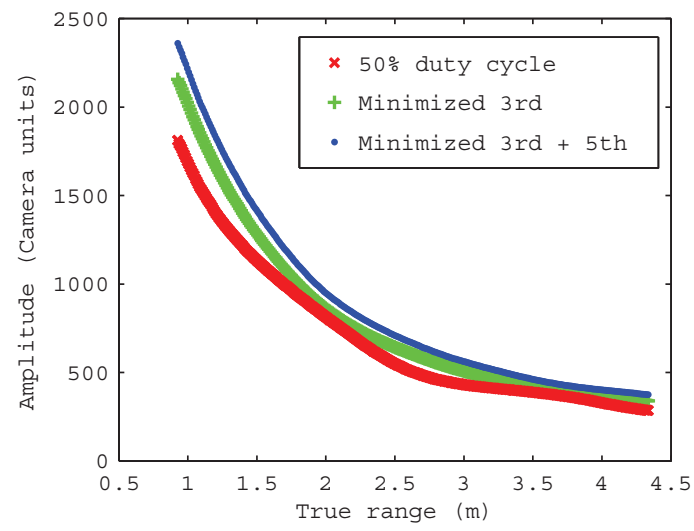

(c)

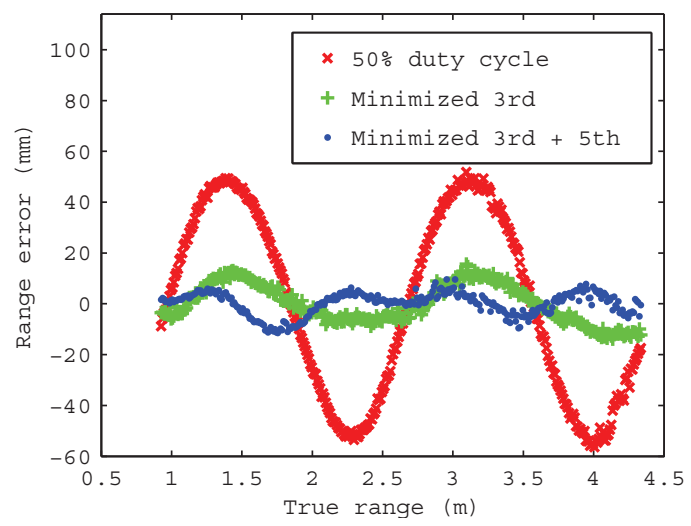

(b)

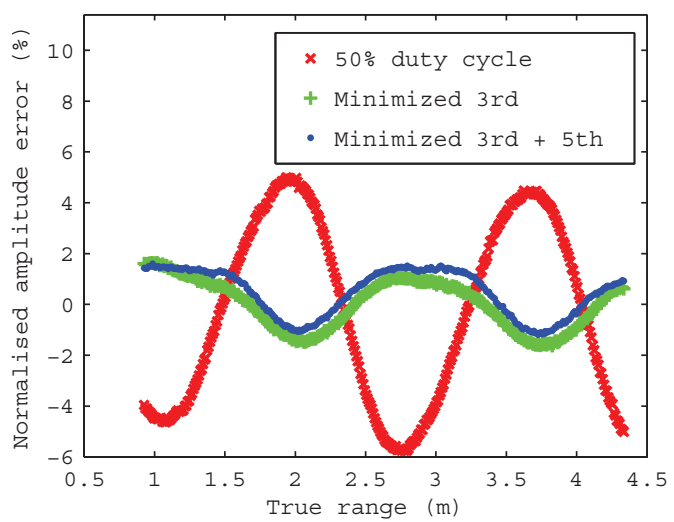

(d)

Figure 9. (a) Three $22 \mathrm{MHz}$ LED modulation waveforms tested; a "standard" $50 \%$ duty cycle (red $\times$ ), a waveform with minimized $3^{\text {rd }}$ order harmonic (green + ), and a waveform with minimized $3^{\text {rd }}$ and $5^{\text {th }}$ order harmonics (blue $\cdot$ ); (b) resultant range linearity error; (c) measured signal amplitude; (d) normalized amplitude error.

Table 1. Parameters and performance results from different LED waveform shapes shown in Figure 9.

\begin{tabular}{|c|c|c|c|c|c|c|}
\hline $\begin{array}{c}\text { Illumination } \\
\text { Waveform }\end{array}$ & $\begin{array}{c}\text { Measured } \\
\text { optical power } \\
\text { (normalized) }\end{array}$ & $\begin{array}{c}3^{\text {rd }} \text { order } \\
\text { harmonic/ } \\
\text { fundamental }\end{array}$ & $\begin{array}{c}5^{\text {th }} \text { order } \\
\text { harmonic/ } \\
\text { fundamental }\end{array}$ & $\begin{array}{c}\text { Linearity error } \\
\text { peak to peak (RMS) }\end{array}$ & $\begin{array}{c}\text { Relative } \\
\text { amplitude }\end{array}$ & $\begin{array}{c}\text { Amplitude error } \\
\text { peak to peak } \\
\text { (RMS) }\end{array}$ \\
\hline $50 \%$ duty cycle & $40.3 \mathrm{~mW}(1.0)$ & $-16.1 \mathrm{~dB}$ & $-27.5 \mathrm{~dB}$ & $108 \mathrm{~mm}(36 \mathrm{~mm})$ & 1.0 & $11 \%(3.6 \%)$ \\
Minimized $3^{\text {rd }}$ & $35.4 \mathrm{~mW}(0.88)$ & $-38.9 \mathrm{~dB}$ & $-25.0 \mathrm{~dB}$ & $30 \mathrm{~mm}(8 \mathrm{~mm})$ & 1.13 & $3 \%(1.0 \%)$ \\
Minimized $3^{\text {rd }}$ and $5^{\text {th }}$ & $38.0 \mathrm{~mW}(0.94)$ & $-33.7 \mathrm{~dB}$ & $-32.6 \mathrm{~dB}$ & $21 \mathrm{~mm}(5 \mathrm{~mm})$ & 1.24 & $3 \%(0.9 \%)$ \\
\hline
\end{tabular}

The measured amplitude values shown in Figure 9 (c) exhibit three characteristics; a decay as range increases, a phase dependent oscillation, and a different magnitude for each waveform. The decay is similar to the inverse-square law, where the amount of light from a point source falling on a target area is inversely proportional to the distance squared. The spatial distribution pattern of the LED illumination defines the shape of this decay curve, and in this instance the captured amplitude is approximately inversely proportional to distance. The decay is constant for all three illumination waveforms, and is therefore not of interest for this study.

An attempt to isolate the amplitude oscillation was performed by taking number of captures at each range, with an electronic phase offset added to each capture. The electronic phase offset introduces a corresponding phase shift in the 
error oscillation. A reference amplitude value at each range can then be produced by averaging captures where the errors are $180^{\circ}$ out of phase, and are therefore cancelled from the measurement. The reference amplitude is subtracted from the original amplitude data, and normalized to remove the decay shape (by dividing by the reference amplitude) to produce the normalized amplitude error shown in Figure 9 (d). A large 11\% peak to peak amplitude error can be seen in the 50\% duty cycle waveform data, which is reduced to approximately $3 \%$ for both of the modified illumination waveforms.

Finally, both of the modified waveforms operated with reduced average optical power compared to the $50 \%$ duty cycle waveform ( $88 \%$ and $94 \%$ respectively), yet both of the modified waveforms also produced a larger amplitude value than the $50 \%$ duty cycle waveform. This effect is due to the increased demodulation contrast resulting from the use of decreased duty cycles, refer section 3 . From the captured data, a relative change in amplitude was calculated by dividing the measured amplitude at each range by the amplitude from the 50\% duty cycle waveform, and then averaging over all values. The modified waveform with minimized the $3^{\text {rd }}$ and $5^{\text {th }}$ order harmonics, on average, had an amplitude $24 \%$ greater than the $50 \%$ duty cycle waveform while only emitting $94 \%$ of the average optical power. This increase in amplitude (demodulation contrast) improves the measurement standard deviation as given by equation (5). To experimentally verify this improvement in measurement precision (standard deviation), a set of 50 range images were captured at each target range, with the mean per-pixel standard deviation shown in Figure 10. The standard deviation values increase approximately linearly with target range due to the decrease in amplitude shown in Figure 9 (c). The waveform with minimized $3^{\text {rd }}$ and $5^{\text {th }}$ order harmonics demonstrated an average standard deviation of 0.83 times that of the $50 \%$ duty cycle waveform.

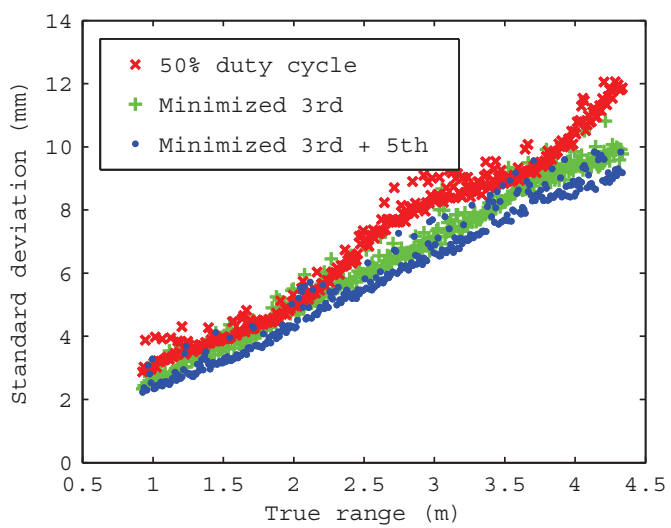

Figure 10. Standard deviation values for the target shown in Figure 8 imaged with different LED illumination waveforms.

An attempt to optimize the LED waveform shape at a modulation frequency of $44 \mathrm{MHz}$ was performed, despite the relatively slow LED rise and fall times compared to those of the laser diode used in section 4 . The results showed that the peak to peak linearity error decreased from $28 \mathrm{~mm}$ to $12 \mathrm{~mm}$, however, for the optimized illumination waveform the systematic error was no longer constrained by the harmonic content of the modulation waveforms. System linearity was instead constrained by another error source that produced two oscillations over a range corresponding to $2 \pi$ radians. Possible causes for this error may include the signal generation circuitry not phase stepping the modulation signals by precisely 90 degrees between captured images, or crosstalk occurring between the LED and image sensor modulation signals. The source of this error is still under investigation.

\section{CONCLUSION}

Reducing the duty cycle of the amplitude modulated illumination waveform, while maintaining the average optical power, is shown to significantly improve both measurement linearity and precision in range imaging cameras. This technique can readily be applied to existing range imaging cameras that use digital circuits to generate and control the amplitude modulated illumination source waveform, and can be adapted for use with either laser or LED illumination. For the standard homodyne 4-sample mode of operation, the optimal illumination duty cycle occurs at approximately $30 \%$, although the exact value is dependent on the harmonic content of the specific image sensor pixel and illumination modulation waveforms. At the optimal duty cycle, the linearity error due to the dominant third and fifth order harmonics can be reduced, while the demodulation contrast can be increased by up to $30 \%$ compared to the typical square wave 
method of operation. The drawback of using this configuration is that the linearity error, while reduced, cannot be completely eliminated and the system will require calibration to remove the residual phase linearity error. The measurement linearity, hence the calibration parameters, are also likely to remain sensitive to system or environmental changes such as temperature fluctuations.

The use of harmonic cancellation provides optimal measurement linearity independent of the illumination duty cycle used; however this comes at the expense of a lower demodulation contrast value than that of the standard homodyne mode (for a given illumination duty cycle). The simultaneous use of a reduced illumination duty cycle and harmonic cancellation is likely to provide the best compromise between measurement linearity and precision as given in Table 2; trading a slight decrease in demodulation contrast for excellent measurement linearity.

Table 2. Performance characteristics of different illumination modulation waveforms.

\begin{tabular}{|c|c|c|c|}
\hline $\begin{array}{c}\text { Light source } \\
\text { configuration }\end{array}$ & $\begin{array}{c}\text { Ease of light source } \\
\text { waveform generation }\end{array}$ & $\begin{array}{c}\text { Demodulation } \\
\text { contrast }\end{array}$ & $\begin{array}{c}\text { Measurement } \\
\text { linearity }\end{array}$ \\
\hline Sine wave & Poor & Poor & Excellent \\
Square wave & Excellent & Good & Poor \\
Optimized duty cycle & Good & Excellent & Good \\
$\begin{array}{c}\text { Harmonic cancellation } \\
\text { with reduced duty cycle }\end{array}$ & Good & Good & Excellent \\
\hline
\end{tabular}

\section{REFERENCES}

[1] Lange, R., "3D Time-of-Flight Distance Measurement with Custom Solid-State Image Sensors in CMOS/CCDTechnology," Ph.D. dissertation, University of Siegen, (2000).

[2] Kahlmann, T. and Ingensand, H., "High-precision investigations of the fast range imaging camera SwissRanger," Proc. SPIE 6758, 67580J (2007).

[3] Boehm, J. and Pattinson, T., "Accuracy of Exterior Orientation for a Range Camera," ISPRS Commission V Mid-Term Symposium 'Close Range Image Measurement Techniques', 103-108 (2010).

[4] Rapp, H., "Experimental and theoretical investigation of correlating TOF-camera systems," Master's thesis, University of Heidelberg, Germany, (2007).

[5] Lindner, M. and Kolb, A., "Lateral and depth calibration of PMD-distance sensors," Advances in Visual Computing, Pt 2, 4292, 524-533 (2006).

[6] Lindner, M., Kolb, A. and Ringbeck, T., "New Insights into the Calibration of ToF-Sensors," IEEE Conf. on Computer Vision and Pattern Recognition (CVPR), 1-5 (2008).

[7] Dorrington, A. A., Cree, M. J., Carnegie, D. A. and Payne, A. D., "Selecting Signal Frequencies for Best Performance of Fourier-based Phase Detection," Proc. Twelfth New Zealand Electronics Conference, 189-193 (2005).

[8] Hsu, S., Acharya, S., Rafii, A. and New, R., "Performance of a Time-of-Flight Range Camera for Intelligent Vehicle Safety Applications," Advanced Microsystems for Automotive Applications 2006, 205-214 (2006).

[9] Dorrington, A. A., Cree, M. J., Carnegie, D. A., Payne, A. D., Conroy, R. M., Godbaz, J. P. and Jongenelen, A. P. P., "Video-rate or high-precision: a flexible range imaging camera," Proc. SPIE 6813, 681307 (2008).

[10] Payne, A. D., Dorrington, A. A., Cree, M. J. and Carnegie, D. A., "Improved Linearity Using Harmonic Error Rejection in a Full-Field Range Imaging System," Proc. SPIE 6805, 68050D (2008).

[11]Büttgen, B. and Seitz, P., "Robust Optical Time-of-Flight Range Imaging Based on Smart Pixel Structures," IEEE Transactions on Circuits and Systems I: Regular Papers, 55, 1512-1525 (2008).

[12] Dorrington, A. A., Carnegie, D. A. and Cree, M. J., "Toward 1-mm depth precision with a solid state full-field range imaging system," Proc. SPIE 6068, 60680K (2006). 
[13] Jongenelen, A. P. P., Carnegie, D. A., Payne, A. D. and Dorrington, A. A., "Maximizing Precision Over Extended Unambiguous Range for TOF Range Imaging Systems," IEEE Instrumentation and Measurement Technology Conference (I2MTC '10), 1575-1580 (2010).

[14] Büttgen, B., "Extending time-of-flight optical 3D-imaging to extreme operating conditions," Ph.D. dissertation, University of Neuchatel, (2006). 